\title{
Residual Stress, Texture, and Phase Investigation of Autogenous Edge Welds Using High Energy Synchrotron Radiation
}

\author{
René V. Martins ${ }^{1,}$, , John A. Francis ${ }^{2, b}$ \\ ${ }^{1}$ Institute for Energy, European Commission, Joint Research Centre, \\ Westerduinweg 3, 1755LE Petten, The Netherlands \\ ${ }^{2}$ The Open University, Walton Hall, Milton Keynes, MK7 6AA, United Kingdom \\ arene.martins@ec.europa.eu, bj.francis@open.ac.uk
}

Keywords: Residual stress, texture, welding, SA508 steel, high energy synchrotron radiation

\begin{abstract}
Two autogenously edge welded beams made from SA508 ferritic steel were investigated with the purpose of validating residual stress modelling tools which are relevant for integrity assessment of structural power plant components. The two specimens were welded with two different torch travel speeds. The residual strain and phase distributions were non-destructively determined by high-energy synchrotron X-ray diffraction. Good agreement between numerical and experimental data was found for the specimen welded at fast speed. Furthermore, the texture changes in the specimen welded at slow speed were analysed by the same experimental technique.
\end{abstract}

\section{Introduction}

The estimation of the residual stress state in structural components is one of the important aspects to be considered in the integrity and lifetime assessment for power plant components. Welded joints of such structures attract particular attention by engineers because of their impact on the microstructure and the significant residual stresses that are generated in and around the fusion zone. Many degradation mechanisms such as stress corrosion cracking [1], creep damage [2], and fatigue cracking [3] can be triggered and/or accelerated by the presence of residual stress. An experimental determination of the stresses is often not possible in thick components, therefore, the integrity assessments are based on simplified bounding residual stress profiles or on Finite Element (FE) modelling results. The validation of such models is often made against experimental data, obtained from benchmarking specimens. As opposed to residual stress modelling for austenitic stainless steel welds, where no phase transformations need to be taken into account, the case of ferritic steels is more complex due to the occurrence of solid state phase transformations. The complexity increases when technically relevant multi-pass welds need to be modelled.

\section{Specimens}

For the purpose of validating constitutive models for material behaviour, autogenously edge welded beams appeared to be an appropriate choice because of the simplicity of the temperature field, which is approximately one-dimensional, and the absence of any filler material, hereby excluding any changes of material behaviour due to compositional changes. Thus, the modelling activities only need to focus on phase transformations and the resulting residual stress field. All specimens were made from SA508 Grade 3 Class 1 reactor pressure vessel steel. The parent material is "ferritic" and typically forms a mixture of bainite and martensite in the weld and heat-affected zone (HAZ) during cooling.

Edge Welds. Two autogenously edge welded beam specimens were manufactured with two different weld speeds. They were produced with the gas-tungsten arc welding process (GTAW) in one pass on the $180 \mathrm{~mm}$ long and $10 \mathrm{~mm}$ wide top edge of the nominally $50 \mathrm{~mm}$ high beam (Fig. 1). The final beam height was larger for the slow weld due to the differences in the resulting weld 
crown geometry. The traverse torch speed for the slow and the fast weld were $1.27 \mathrm{~mm} \cdot \mathrm{s}^{-1}$ and $5.08 \mathrm{~mm} \cdot \mathrm{s}^{-1}$ respectively. In case of the slow weld the specimen was preheated to $150{ }^{\circ} \mathrm{C}$.

$\mathbf{d}_{0}$-Reference and Texture Specimens. $\mathrm{d}_{0}$-reference specimens were produced for both weld specimens. They were manufactured by electro discharge wire cutting from beams welded with the same parameters as the investigated weld specimens. The reference specimens were cut from the through-thickness central position and were rod-shaped with a diameter of $2 \mathrm{~mm}$, and with their long axis parallel to the z-direction. A small notch in the rod in the y-direction allowed recovery of the initial orientation of the rod with respect to the specimen geometry.

The texture specimens were produced in the same way as the reference specimens, but with a diameter of $5 \mathrm{~mm}$ instead of $2 \mathrm{~mm}$, to minimize possible coarse grain effects on the texture measurements. The notch for the recovery of the initial orientation is of particular importance for the texture specimens when tilts and rotations of measured pole figures need to be interpreted with respect to the welding direction.

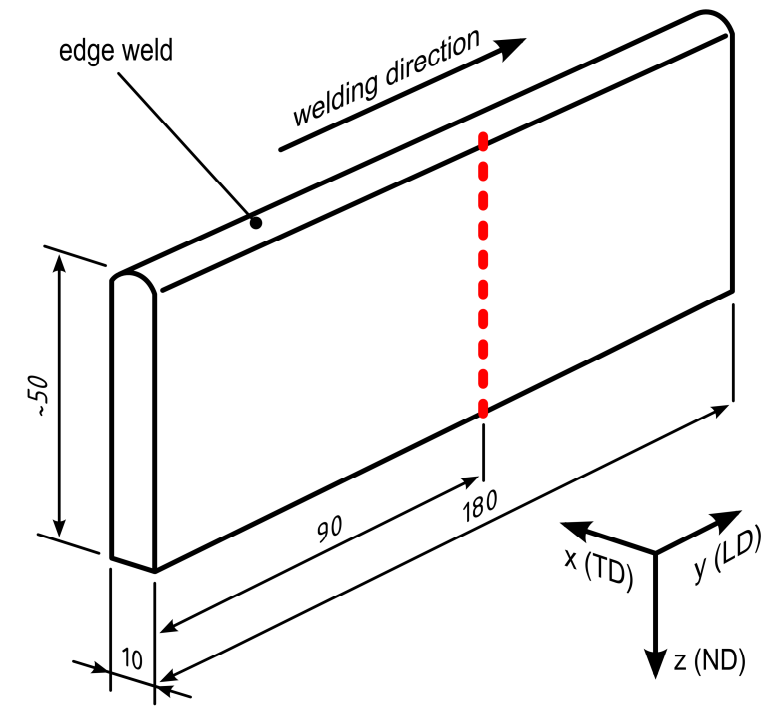

Fig. 1: Schematic representation of edge welded specimen and the coordinate system. TD, LD, and ND denote the transverse, longitudinal, and normal directions respectively. The height, shown as $\sim 50 \mathrm{~mm}$, varied slightly according to the welding speed. The dashed line shows the position of the X-ray diffraction scan line.

\section{Synchrotron X-ray Diffraction Measurements}

The diffraction experiments were performed at the HARWI II beamline at the DORIS III ring of the HASYLAB/DESY, Germany. A view of the setup is shown in Fig. 2. The monochromatic highenergy synchrotron X-rays had a wavelength of $0.01105 \mathrm{~nm}$. The wavelength was determined from a series of diffraction patterns of the Standard Reference Material $660 \mathrm{a}, \mathrm{LaB}_{6}$, purchased from the National Institute of Standards \& Technology. For the strain measurements the beam was confined with apertures to a size of $2.3 \mathrm{~mm} \times 0.25 \mathrm{~mm}(\mathrm{H} \mathrm{x} \mathrm{V})$ at the specimen position. For the texture measurements the beam size was set to $1 \mathrm{~mm} \times 0.3 \mathrm{~mm}(\mathrm{H} \times \mathrm{V})$. The measurements were performed in transmission geometry with the incoming beam parallel to the $\mathrm{x}$-direction. A large area detector was placed $1625 \mathrm{~mm}$ behind the specimen. The detector system used was a MAR345 online image plate scanner. A line in the z-direction was scanned at the specimen mid-length (cf. Fig. 1) with a step size of $\Delta z=0.25 \mathrm{~mm}$ close to the weld and of $\Delta z=2 \mathrm{~mm}$ further away from the HAZ. The corresponding $\mathrm{d}_{0}$-reference specimens were measured with the same experimental setup. They were spun about their z-axis such that approximately one rotation was completed during the exposure time, thus ensuring a homogeneous integration of the signal over almost the entire accessible orientation space. The exposure time was $12 \mathrm{~s}$ per position. The total time was approximately $5 \mathrm{~h}$ for a complete data set, consisting of 78 images for the weld and 78 images for the reference specimen.

Texture measurements were conducted for the slow weld only. Complete diffraction rings up to the austenite $\left\{\begin{array}{lll}3 & 1 & 1\end{array}\right\}$ reflection were recorded with the area detector placed $793 \mathrm{~mm}$ behind the specimen. The cylindrically shaped specimen was rotated about its $z$-axis from $\omega=0$ to $175^{\circ}$ in 
equidistant steps of $\Delta \omega=5^{\circ}$. During exposure the specimen was oscillated about each position $\omega_{\mathrm{i}}$ by $\pm \Delta \omega / 2$, hereby obtaining space-filling and intensity-conserving sampling. The texture measurements were made as a function of distance from the weld top at $16 \mathrm{z}$-positions, in steps of $\Delta \mathrm{z}=0.75 \mathrm{~mm}$ in and close to the weld and at two additional positions in the parent material. The exposure time was $40 \mathrm{~s}$ and the total time for all 16 texture measurements together was about $26 \mathrm{~h}$.

Fig. 2: View of the experimental setup at the HARWI II beamline with the $\mathrm{d}_{0}$ specimen placed in the path of the beam. The specimen-to-detector distance is $1625 \mathrm{~mm}$.

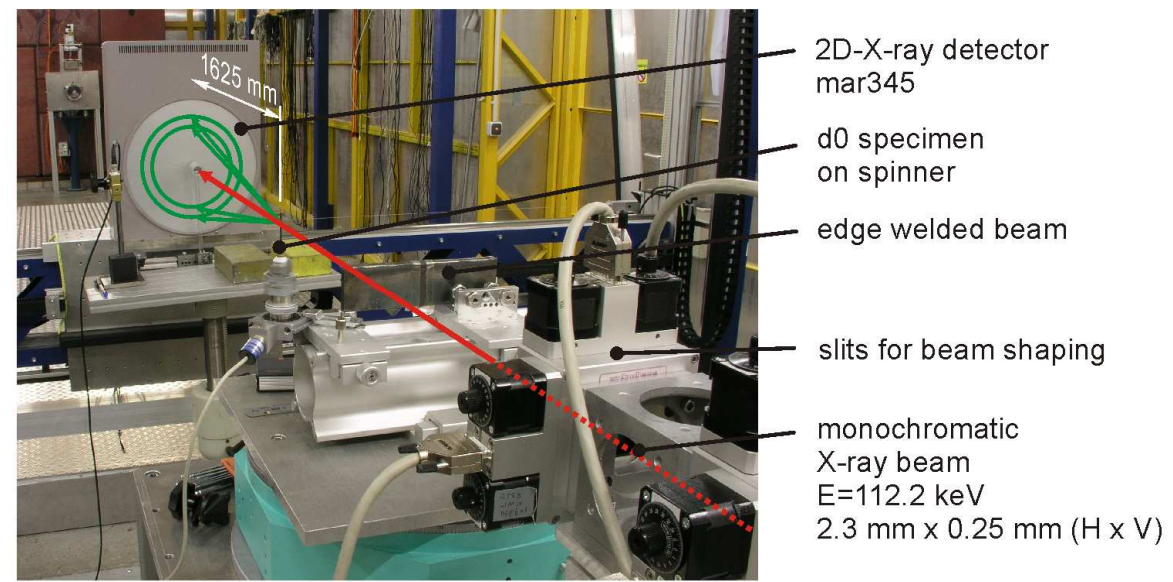

\section{Phase Distributions}

Fig. 3 shows the azimuthally integrated diffraction spectra for the slow and fast $\mathrm{d}_{0}$-reference specimen as a function of the distance from the weld top. Quantitative analyses of the spectra were done by Rietveld refinement using FullProf [4].

The slow weld shows the presence of retained austenite resulting from the welding thermal cycle up to a distance of about $11 \mathrm{~mm}$ from the weld top (Fig. 3a). The maximum austenite fraction of $14 \mathrm{wt} .-\%$ is observed at $8 \mathrm{~mm}$. In the region between 4 and $7 \mathrm{~mm}$ the austenite fraction is $3-4 \mathrm{wt} .-\%$ and between 0 and $3.6 \mathrm{~mm}$ it is on a level of about $7 \mathrm{wt} .-\%$. The phase distribution of the fast

a)

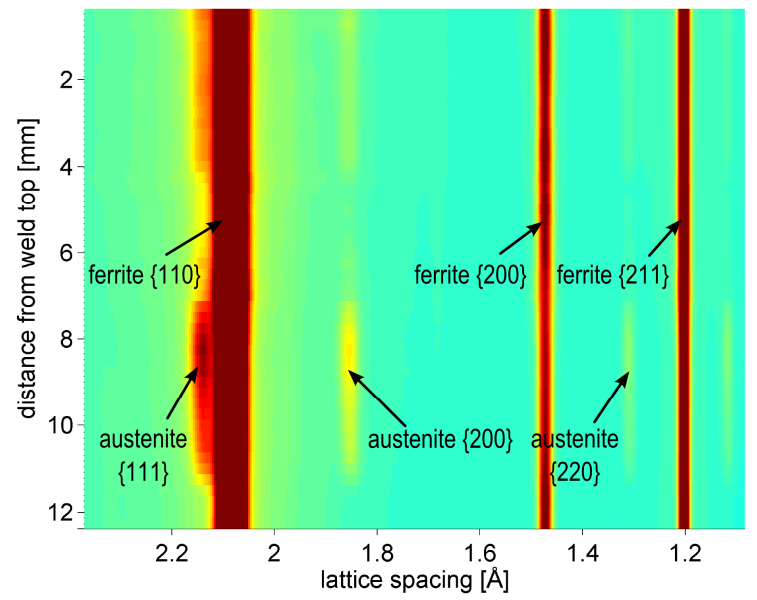

b)

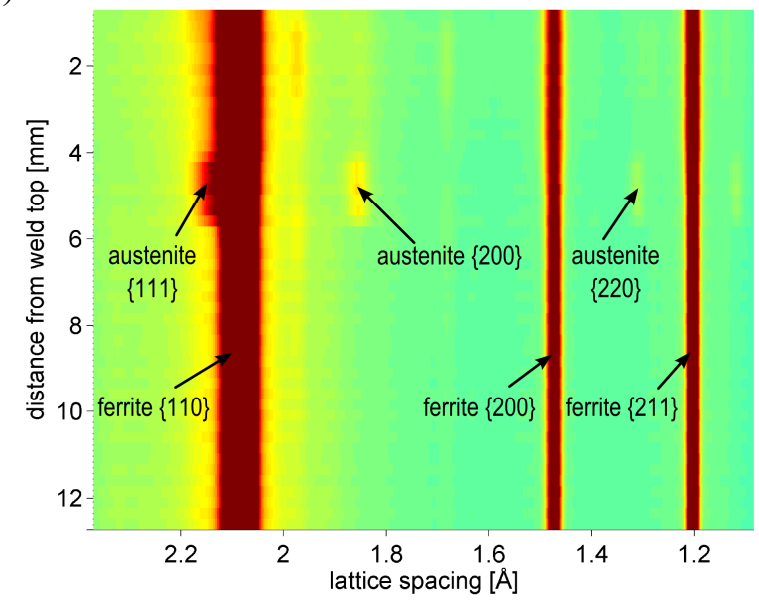

Fig. 3: Diffraction spectra as a function of distance form the weld top surface for the slow (a) and the fast (b) weld specimens.

specimen shows that retained austenite is only present up to $6 \mathrm{~mm}$ from the weld top. The maximum austenite concentration of $9 \mathrm{wt} .-\%$ is observed between 4 and $5 \mathrm{~mm}$. Between 0 and $3.5 \mathrm{~mm}$ the austenite fraction stays on an almost constant level of $3 \mathrm{wt} .-\%$.

In previous investigations of optical micrographs $[5,6]$ three metallographically distinct limits were observed, namely the limit of the fusion zone at $4.25 \mathrm{~mm}$ (slow) $/ 2.75 \mathrm{~mm}$ (fast), the limit of the HAZ at $10.25 \mathrm{~mm}$ (slow) / $6.25 \mathrm{~mm}$ (fast), and a limit dividing the HAZ at $6.75 \mathrm{~mm}$ (slow) / $4.5 \mathrm{~mm}$ (fast). The different extensions of the HAZs into the parent material have their origin in the differences in heat input for the slow and fast weld. For the slow weld specimen, the location of the 
three optically observed limits agrees well with the results from the phase analysis. For the fast weld specimen, the limit between HAZ and parent material seems to fall on the limit of austenite at $6 \mathrm{~mm}$ from the top. However, in this case the phase analysis shows only three different regions, suggesting that the levels of retained austenite in the fusion zone and the upper part of the HAZ are very similar.

\section{Residual Strain Distributions}

Because of the measurement geometry only the strain components in the y/z-plane, integrated over the x-direction, could be measured. The in-plane macro strain tensor components were determined from the distortion, with respect to the corresponding $\mathrm{d}_{0}$-reference specimen, of complete DebyeScherrer rings from the ferrite $\left\{\begin{array}{lll}1 & 1 & 0\end{array}\right\},\left\{\begin{array}{lll}2 & 0 & 0\end{array}\right\}$, and $\left\{\begin{array}{lll}2 & 1 & 1\end{array}\right\}$ diffraction planes. The shear component was in all measurement positions close to zero. The residual stress was calculated by assuming a plane stress state and using elasticity constants that were weighted by the contribution of the individual diffraction planes to the strain tensor values. Fig. 4 shows the results of the measurements and calculations for the slow ( $4 a$ and $c$ ) and the fast weld ( $4 b$ and $d$ ). In addition results from FE modelling are shown in the figure as solid lines. The FE residual stress modelling results were published elsewhere [5], but the corresponding strain values were back-calculated here for the purpose of direct comparison with the experimentally observed in-plane residual strains. hkl weighted elasticity constants were also used for this reverse calculation and the contribution of the austenite phase was not taken into account.

It is obvious from Figs. $4 \mathrm{c}$ and $\mathrm{d}$ that the assumption of plane stress is not correct, despite the small specimen thickness of $10 \mathrm{~mm}$. This is particularly relevant at locations in and close to the weld, where the phase transformations took place. Here the FE model predicts a tensile stress exceeding $250 \mathrm{MPa}$ in the transverse direction (i.e. the direction not accessible for the diffraction measurements here) for the fast weld, and up to $100 \mathrm{MPa}$ for the slow weld. Further away from the weld the plane stress assumption is again valid.

The comparison of modelled and measured strains shows much better agreement, particularly for the fast weld where the strain magnitudes agree well and the locations of minima and maxima are only shifted by about $1 \mathrm{~mm}$. This shift could be attributed to small local variations of the limits between fusion zone, HAZ, and parent material.

In the case of the slow weld specimen the measurement and modelling results do not agree as well. The magnitudes of the longitudinal strain maxima match well, but large differences in excess of $1000 \mu \varepsilon$ are observed within the first $10 \mathrm{~mm}$ below the weld top. Also, there was no experimental evidence for the negative slope of the longitudinal strain towards the bottom of the specimen. While the slight shift of the longitudinal strain peak location could be again explained by small variations of the depth of metallurgically different zones, the larger differences in the magnitude and shape of the strain distribution might have their origin in an incorrect prediction of the phase concentrations or, alternatively, they may be related to a shortage of phase-dependent material property data.

Overall, in both specimens the maximum longitudinal strain values are observed about 1 to $2 \mathrm{~mm}$ beneath the zone with the highest austenite concentration. The presence, in the longitudinal direction, of a compressive zone inside the fusion zone and HAZ, and of a tensile zone just outside the HAZ can be explained by the occurrence of solid-state transformations and the transformation temperatures of SA508 steel $[6,7]$. During welding SA508 transforms from ferrite to austenite upon heating, and upon cooling to a bainite/martensite mixture with a fraction of retained austenite, which varies with depth. The depth up to which the austenitisation temperature is reached is greater for the slow weld than for the fast weld. During cooling the thermal contraction strain is compensated by the transformation strain associated with the formation of bainite and martensite. When, as in the present case, the transformation temperature is sufficiently low, this can lead to an over compensation, such that compressive longitudinal stresses remain in the weld zone and HAZ, and tensile longitudinal stresses build up in the adjacent base material. 
a)

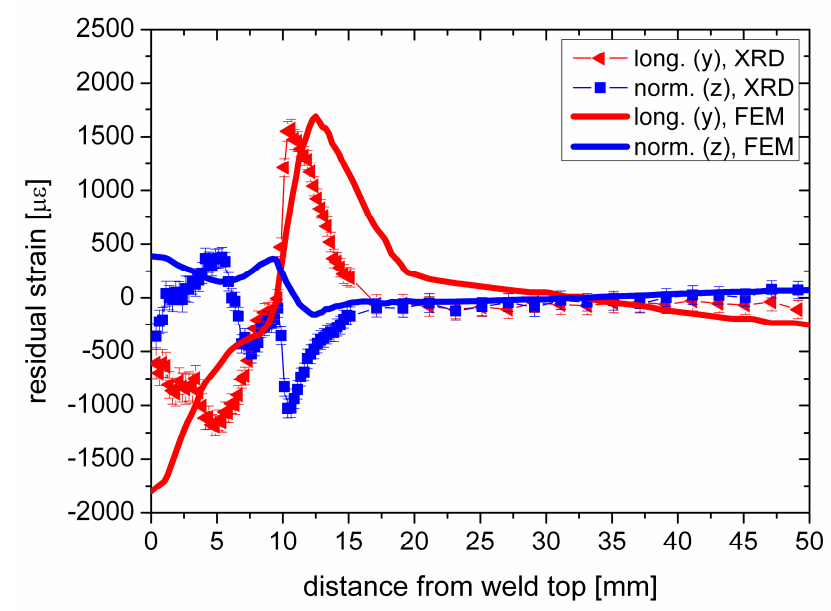

c)

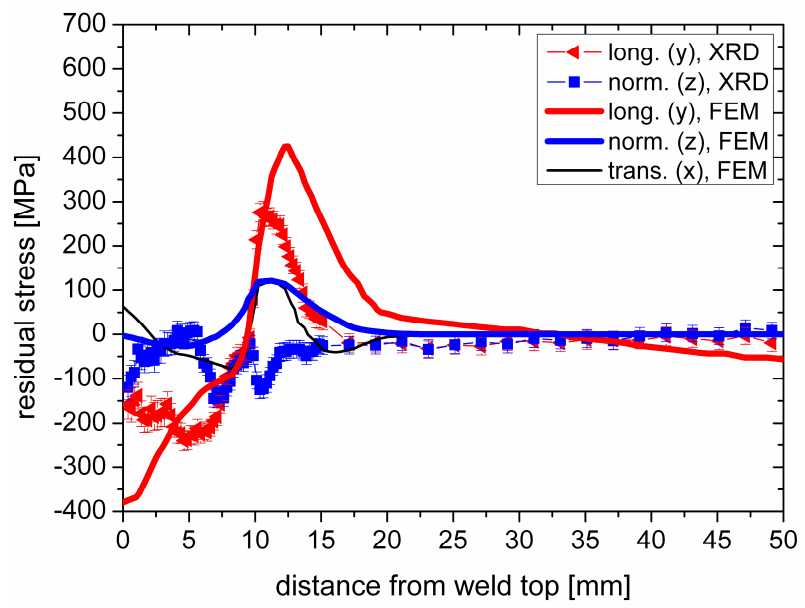

b)

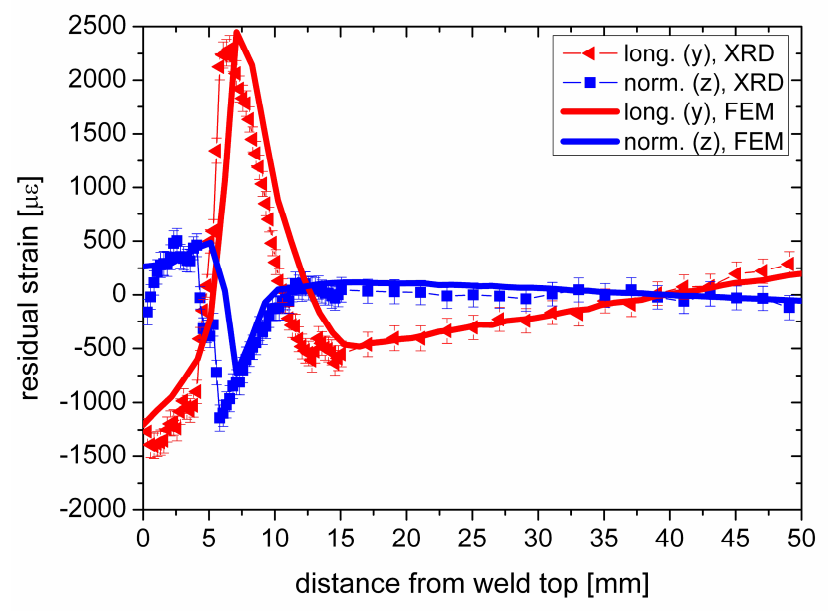

d)

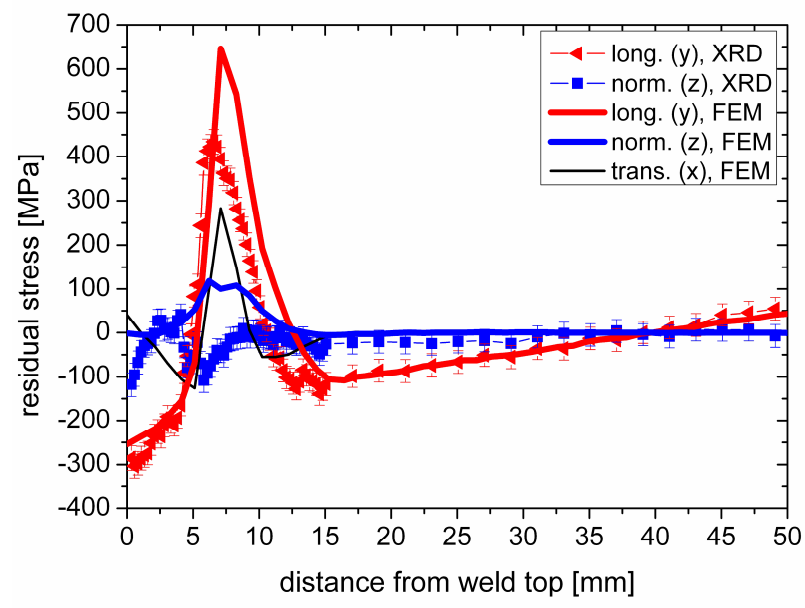

Fig. 4: Residual strain and stress distributions for slow (a and c) and fast (b and d) welding speeds. Stresses were calculated assuming plane stress. FE modelling macroscopic stress results, as published in [5], are plotted as solid lines. FE modelling strain results were calculated from the stress values. FE modelling transverse strain component was not plotted for better readability.

\section{Texture in the Fusion Zone}

The Orientation Density Functions (ODF) for the different positions were calculated based on almost complete experimental ferrite $\left\{\begin{array}{lll}1 & 1 & 0\end{array}\right\},\left\{\begin{array}{lll}2 & 0 & 0\end{array}\right\}$, and $\left\{\begin{array}{lll}2 & 1 & 1\end{array}\right\}$ pole figures. The MTEX toolbox [8] was used to perform all texture related calculations. In general, the texture indices observed at the different distances from the weld top were all rather weak, with maximum values well below 2 . The texture index in the parent material was, with a value of 1.06 , close to random texture.

Fig. 5a shows a first result from the texture analyses at a distance of $0.925 \mathrm{~mm}$ from the weld top surface, i.e. in the fusion zone of the weld. The ferrite $\left\{\begin{array}{lll}1 & 1 & 0\end{array}\right\}$ pole figure was recalculated from the corresponding ODF. The welding direction points out of the plane and the weld transverse and normal directions are indicated by TD and ND respectively. The texture index calculated at this position is 1.37. A clear fibre texture is observable. The fibre axis is tilted away from the normal direction in the transverse plane by about $27^{\circ}$ and, in the longitudinal-normal plane, away from the normal direction, in opposite sense to the welding direction, by about $7^{\circ}$. At a position $0.75 \mathrm{~mm}$ further away from the weld top, i.e. at $1.675 \mathrm{~mm}$, the texture index diminishes to 1.11 and the fibre texture is only weakly observable (Fig. 5b) with a tilt now of about $5^{\circ}$ in opposite sense to the welding direction. 
Fig. 5: Ferrite $\left\{\begin{array}{lll}1 & 1 & 0\end{array}\right\}$ polefigure at a distance of $0.925 \mathrm{~mm}$ (a) and $1.675 \mathrm{~mm}$ (b) from the weld top. The welding direction points out of the plane, weld transverse (TD) and normal (ND) directions are indicated.

The pole figures were recalculated from the corresponding ODF. a)

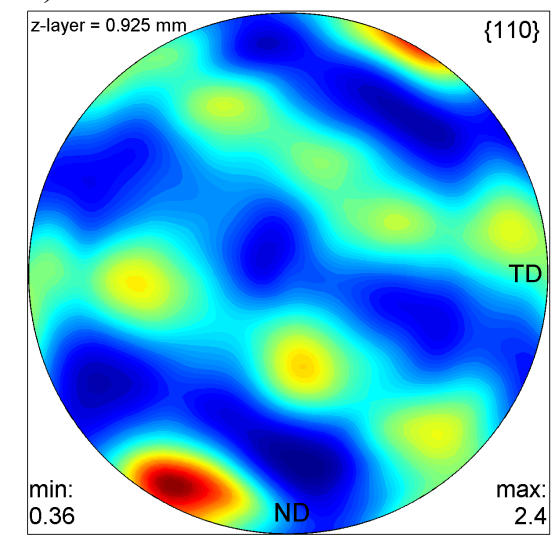

b)

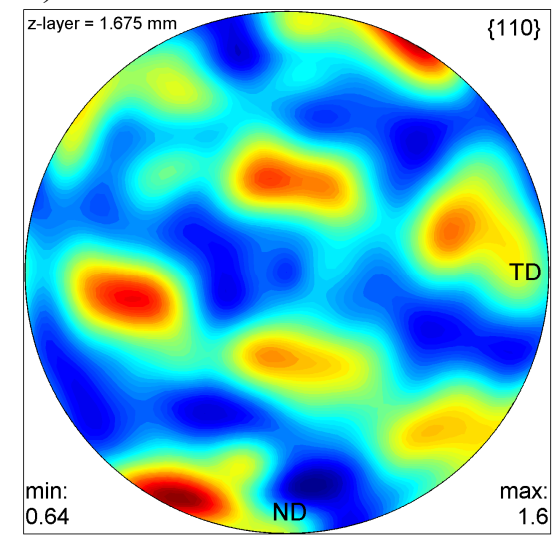

\section{Conclusions}

Non-destructive high-energy X-ray diffraction studies have been conducted on autogenous edge weld beams. The specimens were made from SA508 ferritic steel and were welded with two different traverse speeds, with one being four times higher speed than the other. The experimental results show a strong link between the phase concentrations and the residual strain distributions. In both specimens the highest tensile longitudinal strain was observed just outside the HAZ. In the lower part of the HAZ (further away from the fusion zone) the maximum austenite fractions were found. In the fusion zone the austenite fractions were about $40 \%$ of the respective maxima in the lower HAZ. In addition, the slow weld exhibited in the upper part of the HAZ a region with an austenite fraction of $20 \%$ of the maximum value. In general, the austenite fractions in the slow weld were a factor of about 1.5 to 2 higher than in the fast weld. The agreement between experiment and data from a previously published FE model is good for the fast weld. For the slow weld the agreement is less convincing, which might have its origin either in the modelling of the phase proportions, or in the shortage of the phase-dependent material property data that is required by FE models. First results from texture measurements show a weakly developed fibre texture in the first $1.8 \mathrm{~mm}$ from the weld top. In general, the observed texture indices are all well below 2 .

\section{Acknowledgements}

The authors acknowledge the allocation of beamtime at HASYLAB/DESY and the support of the HARWI II beamline staff. They acknowledge the NeT partners for making specimens and material available and for sharing their results.

\section{References}

[1] D.J. Hornbach, P.S. Prevey: J. Press. Vess.-T ASME, 124, 3, (2002) 359-365.

[2] M. Turski, P.J. Bouchard, A. Steuwer, P.J. Withers: Acta Mater., 56, 14 (2008), 3598-3612.

[3] W. Fricke: Materialwiss. Werkst., 36, 11 (2005), 642-649.

[4] J. Rodriguez-Carvajal: Physica B, 192 (1993), p. 55.

[5] A. P. Warren, I. Symington, S.K. Bate, J.A. Francis, M. Turski, P.R. Hurrell: Proc. PVP2008, Vol. 6 Pt. A and B, Paper 61509, ASME, pp. 517-525, Published 2009.

[6] J.A. Francis, M. Turski, P.J. Withers: Proc. PVP2008, Vol. 6 Pt. A and B, Paper 61469, ASME, pp. 459-466, Published 2009.

[7] J.A. Francis, H.K.D.H. Bhadeshia, P.J. Withers: Mater. Sci. Tech.-Lond., 23 (2007) 1009-1020.

[8] R. Hielscher, H. Schaeben: J. Appl. Crystallogr., 41 (2008) 1024-1037. 